\title{
GLOBAL PHASE BEHAVIOUR OF BINARY MIXTURES OF LENNARD-JONES MOLECULES
}

\author{
V.A. MAZUR, L.Z. BOSHKOV \\ Technological Institute of Refrigerating Industry, I/3 Petra, Velikogo St., 270000 Odessa, USSR \\ and \\ V.G. MURAKHOVSKY ${ }^{1}$ \\ Inorganic Chemistry Department, State University of Utrecht, Croesestraat 77A, 3522 AD Utrecht, The Netherlands
}

Received 26 April 1984

Revised manuscript received 6 July 1984

\begin{abstract}
The one-fluid model of equation of state and thermodynamic description of boundary states are used to study global equilibrium surface of two-component Lennard-Jones fluids. The global phase diagram for equal size molecules with seventeen separate regions is reported. The new type of phase behaviour is distinguished.
\end{abstract}

The great variety of phase behaviour which was found for binary fluid mixtures is conditioned by intermolecular interaction. The successive theoretical approach to the analysis of the observed phase phenomena in terms of microscopic characteristics is usually restricted by the models of lattice gas in the local regions of the thermodynamic equilibrium surface. The global analysis of the equilibrium surface of coexisting fluid phases was reported only for the binary van der Waals mixtures $[1,2]$. The topological structure, created by the one-fluid van der Waals model, is of such a kind that it reflects many qualities of phase behaviour which were discovered by means of the binary systems that were already studied experimentally. There are, however, some reasons to require this model to be defined more exactly. In the first place, there is no direct correlation between the equation of state parameters and the characteristics of the intermolecular potentials; secondly, essential quantitative discrepancy in predicting the properties for the given binary mixtures is observed; thirdly, the true asymptotic behaviour of the equation of state at the high density limit is not realized. Among more

1 Permanent address: Physical Department, Technological Institute of Refrigerating Industry, 1/3 Petra Velikogo St., 270000 Odessa, USSR. realistic models of mixtures, in which the faults, mentioned above, can be eliminated, the most explored one is the binary fluid mixture of Lennard-Jones (LJ) molecules. We have presented the equation of state for the mixture of LJ molecules in Ree form [3] in the well-known [4] one fluid approximation with the potential parameters of the mixture $\epsilon \sigma^{3}$ and $\sigma^{3}$ quadratically dependent on the mole fractions of the components. The expression for the molar Helmholtz free energy of a binary $\mathrm{LJ}$ mixture has the form:

$$
\begin{aligned}
f & =\sum_{i=1}^{4} B_{i} y^{i} / i+B_{10} y^{10 / 10}-\left(T^{*}\right)^{-1 / 2} \sum_{i=1}^{5} C_{i} y^{i} \\
& +\left(T^{*}\right)^{-1} \sum_{i=1}^{5} D_{i} y^{i} / i+f_{0},
\end{aligned}
$$

where

$$
\begin{aligned}
f_{0} & =-\ln v+x \ln x+(1-x) \ln (1-x) \\
& +x \psi_{2}(T)+(1-x) \psi_{1}(T),
\end{aligned}
$$

$B_{i}, C_{i}, D_{i}$ are the coefficients of the Ree equation [3], $y=\rho^{*} /\left(T^{*}\right)^{1 / 4}, \rho^{*}=N_{\mathrm{A}} \sigma^{3} / v, T^{*}=k T / \epsilon, v:$ molar volume of the mixture, $k$ : Boltzmann constant, $N_{\mathrm{A}}$ : Avogadro's constant, $T$ : temperature.

This paper presents the results of the study of the 
global equilibrium surface for the two-component $\mathrm{LJ}$ fluid. The global equilibrium surface is the result of applying the thermodynamic conditions, corresponding to the description of the coexisting phases, critical states and some other features in the space of the field variables $S \times Z$, where $S$ is the space of the state parameters, which are the same in all phases; $Z$ is the space of parameters for the regarded model of the equation of state:

$z_{1}=\left(\epsilon_{22}-\epsilon_{11}\right) /\left(\epsilon_{22}+\epsilon_{11}\right)$,

$z_{2}=\left(\epsilon_{22}-2 \epsilon_{12}+\epsilon_{11}\right) /\left(\epsilon_{22}+\epsilon_{11}\right)$,

$z_{3}=\left(\sigma_{22}-\sigma_{11}\right) /\left(\sigma_{22}+\sigma_{11}\right)$,

$z_{4}=\left(\sigma_{22}-2 \sigma_{12}+\sigma_{11}\right) /\left(\sigma_{22}+\sigma_{11}\right)$.

The following phenomena: (1) coexistence of two phases; (2) critical states; (3) three-phase equilibrium; (4) azeotropic lines; (5) specific points of equilibrium surface - the critical and the azeotropic end points, the critical azeotropic state, the equilibrium points of four fluid phases - these completely describe the sections of the global equilibrium surface, given by the mapping $\psi: \mathrm{S} \times \mathrm{Z} \rightarrow \mathrm{S} \times\{z\}, z \in \mathrm{Z}$ and make it possible to carry out their classification [2].

To study more general properties of the global equilibrium surface, we have considered the boundary states, corresponding to the transition of one phase behaviour type to the other and have described them thermodynamically. The boundary state, characterizing the transition between types III and IV (by the van Konynenburg and Scott classification [2]) is presented by the double critical end-point (DCEP). The thermodynamic description of the DCEP is:

$f_{1}=p^{(1)}-p^{(2)}=0, \quad f_{2}=\mu_{1}^{(1)}-\mu_{1}^{(2)}=0$,

$f_{3}=\mu_{2}^{(1)}-\mu_{2}^{(2)}=0, \quad f_{4}=\left(\partial^{2} g / \partial x^{2}\right)_{p, T}^{(1)}=0$,

$f_{5}=\left(\partial^{3} g / \partial x^{3}\right)_{p, T}^{(1)}=0$,

$f_{6}=\frac{D\left(f_{1}, f_{2}, f_{3}, f_{4}, f_{5}\right)}{D\left(x^{(1)}, v^{(1)}, T, x^{(2)}, v^{(2)}\right)}=0$,

where $\mu_{j}^{(i)}$ are the chemical potentials of component $j$ in phase $i, g$ : molar Gibbs free energy, $p, T, v, x$ : pressure, temperature, molar volume and mole fraction of second components, respectively, $f_{6}$ : jacobian of the set of equations $f_{1}-f_{5}$. The boundary between the types I and V, I-A and V-A, II and IV, III and IV-i, III-A and IV-A is determined by the description of tricritical points. In the tricritical point of the two-component system the second, third, fourth and fifth Gibbs free energy derivatives as to their composition are equal to zero:

$$
\begin{aligned}
& \left(\partial^{2} g / \partial x^{2}\right)_{p, T}=\left(\partial^{3} g / \partial x^{3}\right)_{p, T}=0, \\
& \left(\partial^{4} g / \partial x^{4}\right)_{p, T}=\left(\partial^{5} g / \partial x^{5}\right)_{p, T}=0 .
\end{aligned}
$$

On transition of the systems with azeotropy to the systems without azeotropy the boundary state is given by the critical azeotropic point on the stabilitymetastability boundary, on one side, i.e. by the critical azeotropic phase in coexistence with the normal phase; and on the other side, by the critical azeotropic states coinciding with the critical points for the pure components. Another boundary state is the point of coexistence of three phases, one of which is the critical phase.

From the mathematical point of view, the determination of the boundaries between the types is equivalent to searching out all the roots of the systems of nonlinear algebraic equations. However, the lack of a priori information concerning the solution structure makes the numerical analysis for nontrivial models of the equation of state considerably more complicated. The constructive approach which combines generalization and reliability lies in formulating the thermodynamic models as the problems of multiextrema of nonlinear programming [5]. The simplified example for DCEP is:

$\min _{y \in Y} F(y)\left\{F\left(x^{(1)}, v^{(1)}, T, x^{(2)}, v^{(2)}, z_{2}\right)=\Sigma_{i=1}^{6} f_{i}^{2}\right\}$, where $f_{i}$ are the functions comprising the thermodynamic description of DCEP.

The projection $\Pi_{z}: S \times Z \rightarrow Z$, converts the global equilibrium surface into the global phase diagram, one section of which is shown in fig. 1 . This section corresponds to the case of equal size molecules, i.e. $z_{3}=z_{4}$ $=0$. The continuous lines separate the regions of the energetic characteristic values, corresponding to different types of phase behaviour. The locus of the boundary lines is determined exactly by solving respective sets of equations with the aid of a computer. In the case considered here, $\sigma_{11}=\sigma_{12}=\sigma_{22}$, we can mark out seventeen types of phase behaviour. Their position in the global phase diagram (fig. 1) is not equivalent. 


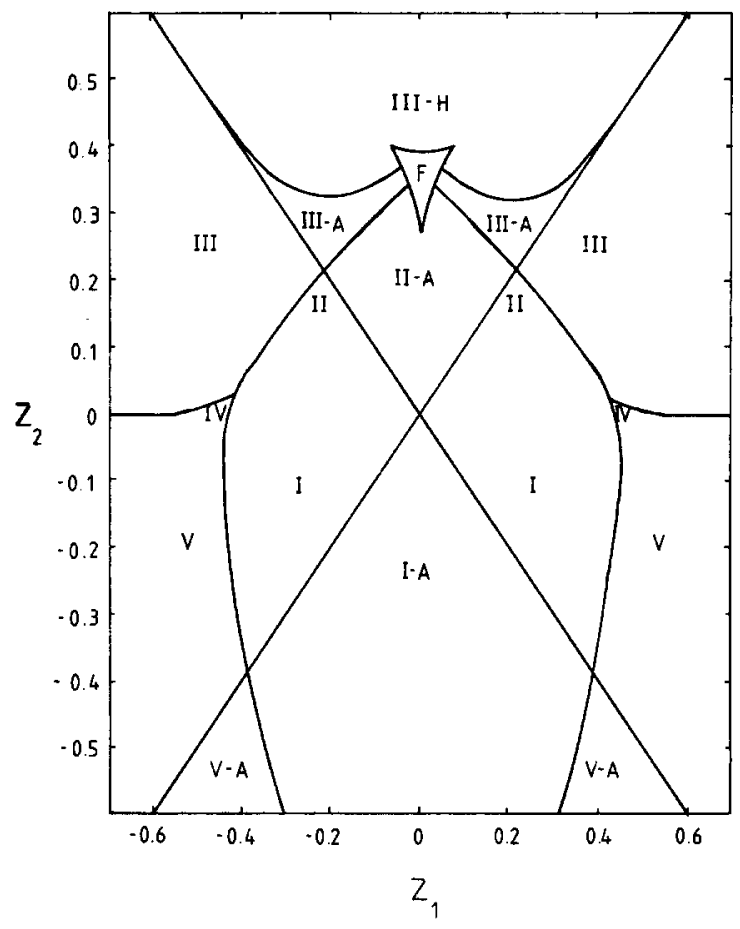

Fig. 1. Global phase diagram for binary mixtures of equal size Lennard-Jones molecules.

Thus, the IV-I and IV-A types occupy a very narrow region between II and III, II-A and III-A respectively, and they are not shown in fig. 1. The boundaries between the types I and II and the other pair of types, $\mathrm{V}$ and IV are not shown as well, because there is no boundary state, characterizing these transitions, when the existence of the solidification line is ignored.

The analysis of the global equilibrium surface made it possible to mark out the III-A type region, which has not been described previousiy in the literature (fig. 2). The systems with phase behaviour of this type are characterized by two critical lines, one of which connects the critical point of the first component $C_{1}$ with the critical end-point $C_{E}$ on the three-phase line, when passing through the critical azeotropic point, $\mathrm{C}_{\mathrm{A}}$; the second critical line begins at the critical point of the second component $C_{2}$ and goes away to the high pressure region. A positive azeotrope is observed in the restricted range from the azeotropic end-point $A_{E}$ on the three phase line to $C_{A}$.

One can expect the display of phase behaviour for the III-A type in the real two-component systems,

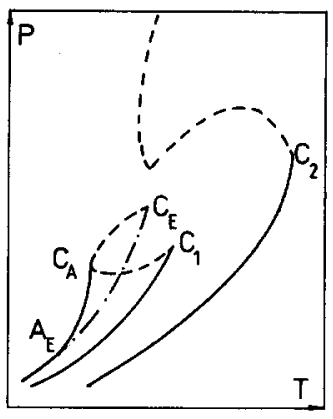

Fig. 2. Schematic $p, T$ projection of II-A phase behaviour type.

since a greater part of the types discovered have analogues among the systems that were already studied experimentally.

There is a region $-F$, shown in fig. 1 , which is analogous to the "shield" region discovered by Furman and Griffits [1] for binary van der Waals mixtures, for which the equilibrium of four fluid phases is possible. Schematically it is shown in fig. 3, where five types of phase behaviour, possible in this region, are marked out. Three tops of it correspond to the tricritical points, coexisting with normal phases, and boundaries determine the points of coexistence of the critical phase with two normal ones. The lines of tricritical and those of double critical end-points run in the F-region so close to each other that the difference between them can be neglected. Thus we do not distinguish here the types, corresponding to this very narrow band, which serves as continuation to the region IV-A.

The topological features of the global equilibrium

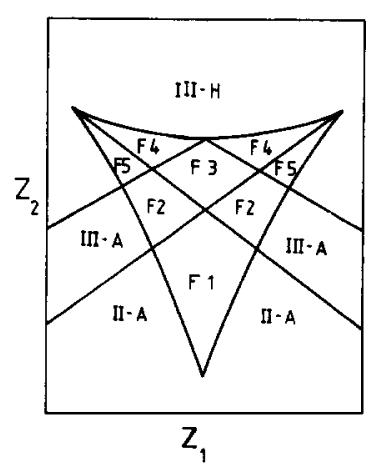

Fig. 3. Region $F$ in global phase diagram (schematically). 
surface for the one-fluid van der Waals model and the one-fluid model of $\mathrm{LJ}$ binary fluid mixture in many respects coincide, but the boundary relations of the parameters for the LJ fluid are more realistic. Comparison of the equations of state indicates the existence of a correlation of phase behaviour types, not only with relation to the critical parameters of the pure components, but also with relation to the saturation lines on the whole, - besides not only for the case of close critical parameters of the components.
References

[1] D. Furman and R.B. Griffits, Phys. Rev. A17 (1978) 1139.

[2] P.H. van Konynenburg and R.L. Scott, Phil. Trans. Roy. Soc. A298 (1980) 495.

[3] F. Ree, J. Chem. Phys. 73 (1980) 5401.

[4] J.S. Rowlinson, Liquids and liquid mixtures, 2nd Ed. (Butterworth, London, 1969) ch. 9.

[5] L.Z. Boshkov and V.A. Mazur, in: The application of mathematical methods for the description and study of phys.-chemistry equilibriums (Irkutsk, USSR, 1982) p. 128. 